\title{
Statistical analysis of Salmonella test data and comparison to results of animal cancer tests
}

\author{
Joyce McCann, Lois Swirsky Gold, Laura Horn, R. McGill ${ }^{1}$, T.E. Graedel ${ }^{1}$ \\ and John Kaldor * \\ Lawrence Berkeley Laboratory, Berkeley, CA, and 'AT and T Bell Laboratories, Murray Hill, NJ (U.S.A.)
}

(Received 20 March 1987)

(Revision received 2 October 1987)

(Accepted 9 October 1987)

Keywords: Salmonella test data, statistical analysis; Animal cancer tests.

\section{Summary}

A quantitative framework for the analysis of results of the Salmonella (Ames) test is presented, and the relationship between mutagenesis and carcinogenesis is examined. Color graphics are used for the Salmonella data to describe variability, and trends across multiple chemicals and test conditions. Positivity in the Salmonella test, using statistical criteria to classify results, is compared to positivity in carcinogenesis bioassays for 48 chemicals tested in NCI/NTP-sponsored programs. Sensitivity of the Salmonella test across 5 tester strains was 91\% (21/23), while specificity was only 36\% (9/25). Results were most concordant for TA100 Aroclor-induced rat S9: sensitivity was $87 \%$, specificity $64 \%$. The correlation of mutagenic potency and carcinogenic potency was $0.41(p<0.001)$ for 80 chemicals, using results from both the general published literature and the NCI/NTP-sponsored programs. After removal of 3 extreme values, the correlation was $0.24(p=0.04)$.

It would be advantageous to utilize more fully the large number of short-term tests for genotoxicity to aid in the extrapolation of experimental data to humans. In spite of the increased use of such tests (e.g., see Hollstein et al., 1979; IARC, 1980), their ability to predict carcinogenic potential has remained controversial and uncertain. In 1975, we reported encouraging results from tests of some

Correspondence: Dr. Joyce McCann, ICF/Clement Associates, Inc., 160 Spear St., 13th Floor, San Francisco, CA 94105 (U.S.A.).

\footnotetext{
* Current address: International Agency for Research on Cancer, Lyon (France).
}

300 carcinogens and non-carcinogens in the Salmonella (Ames) test (McCann et al., 1975, 1976. Meselson and Russell (1977) affected the course of research in the field by publishing a startlingly high correlation between mutagenic potency in the Salmonella test and carcinogenic potency in rodent carcinogenesis tests. Clive et al. (1977) reported a similarly high correlation for the mouse lymphoma assay shortly thereafter.

There has been spirited and unresolved controversy about the relationship between mutagenesis and carcinogenesis. Failure to reach consensus is due largely to our limited understanding of the mechanisms of carcinogenesis, and our consequent inability to construct critical experimental 
paradigms. It is not even clear that animal cancer tests are an adequate standard against which to measure the utility of short-term tests for prediction of human cancer. In addition, the mutagenesis data sets have frequently been small or confined to a few or a single chemical class. Statistical procedures for estimating potency and determining positivity have rarely been used, nor have criteria been included to insure the adequacy of data. Studies have also been limited by the absence of short-term test data on non-carcinogens.

We describe here our efforts to develop a quantitative framework in which to analyze results of the Salmonella test and to examine the relationship between mutagenesis and carcinogenesis.

\section{Methods}

\section{(A) Sources of data}

Mutagenesis data are from a data tape supplied to us by NCI containing data from an NCI/ NTP-sponsored Salmonella testing program (Dunkel et al., 1984, 1985) and are from the general published literature (McCann et al., 1984). Carcinogenesis data are from the NCI/NTP Bioassay Program and the general published literature as reported in the Carcinogenic Potency Database (CPDB) (Gold et al., 1984). Briefly, the CPDB organizes the long-term carcinogenesis literature, provides estimates of carcinogenic potency for several hundred compounds, and displays results in a plot format. All experiments in the database meet a set of inclusion criteria, e.g., the test agent was administered alone rather than in combination with other substances; the protocol included a control group; the route of administration of the test agent was either diet, water, gavage, inhalation, intravenous injection or intraperitoneal injection; the length of experiment in rodents was at least 1 year with dosing for at least 6 months.

\section{(B) Positivity}

For the comparison in positivity between Salmonella tests and carcinogenesis bioassays, we have used closely matched tests of 48 substances from the NCI testing programs cited above. This number is less than the total number of chemicals tested in these programs because we required that: carcinogenesis data be restricted to those results in the CPDB as of 1984 (Gold et al., 1984); each chemical have adequate carcinogenesis tests in both sexes of $\mathrm{B} 6 \mathrm{C} 3 \mathrm{~F} 1$ mice and both sexes of Fisher 344 rats; and each chemical be tested in 20 Salmonella protocols [induced and uninduced S9 from B6C3F1 mice and Fisher 344 rats; 5 tester strains (TA100, TA98, TA1535, TA1537, TA1538)]. Animal tests and Salmonella assays were both conducted using the same batch preparations of each chemical, and the same mouse and rat strains were used in the cancer test and as the liver homogenate in the Salmonella test. Data from the published literature were not utilized because the chemicals had not all been tested under the same protocols or to the same degree of thoroughness, thus possibly resulting in incorrect classifications. In addition, negative results are often not published, which would also have biased the data-set.

Carcinogenesis. To classify a chemical as carcinogenic in this analysis, we have used the results of all 4 experiments in an NCI/NTP bioassay: male rats, female rats, male mice, and female mice. A chemical is classified as positive if any single experiment was positive. For each experiment we use the evaluation in the NCI/NTP Technical Report, since evaluations of carcinogenicity often take into account more than statistical significance, e.g., historical control rates, survival and latency, and/or response. Each experiment is categorized as positive if the NCI/NTP evaluation was clear evidence of carcinogenicity, as negative if the evaluation was no evidence of carcinogenicity, and as associated with carcinogenicity if there was suggestive or equivocal evidence. Generally, this designation of positivity corresponds well with the statistical significance associated with testing whether the slope of the dose response is different from zero (Gold et al., 1984).

Mutagenesis. A test for statistical significance of individual mutagenesis experiments was previously described (Bernstein et al., 1982). The positivity of replicated experiments was determined using a summary $p$ value (McCann et al., 1984). A set of replicate experiments was considered positive overall if the summary $p$ value was less than 
0.05 and, for the NCI/NTP data, if at least 2 experiments in the replicate data-set were positive $(p<0.05)$. The positivity of a chemical tested under different test conditions was determined if the statistical significance of any summary $p$ value was less than $1-(1-0.05) 1 / k$, where $k$ is the number of test conditions (Fisher, 1958).

\section{(C) Estimation of potency}

For the comparison of carcinogenic and mutagenic potencies, we have used all results from two large databases, covering data from both the general literature and NCI/NTP testing programs. All chemicals were selected that had a positive result in both the mutagenesis database and the carcinogenesis database.

Carcinogenesis. A compound is categorized as a carcinogen for the potency analysis if the author's opinion for an experiment in the CPDB was positive. Tests for which the evaluation was only "associated" or "suggestive" have not been included. From among all chemicals in the mutagenic and carcinogenic potency databases, 80 compounds were identified as both mutagens and carcinogens, and these constitute the data set for the correlation. The CPDB contains estimates of carcinogenic potency using the index $\mathrm{TD}_{50}$ (Sawyer et al., 1984; Peto et al., 1984; Gold et al., 1984). Briefly, $\mathrm{TD}_{50}$ is that daily dose rate in $\mathrm{mg} / \mathrm{kg}$ body $\mathrm{wt} /$ day which would induce tumors in half the test animals that would have remained tumor-free at zero dose. The calculation standardizes the results of rodent experiments by taking into account the spontaneous tumor rate, using lifetable data when available, and adjusting for early termination of dosing or experiment length before 2 years. Our standard values for animal weight, intake of food, air, and water, and standard lifespans are given in Gold et al. (1984). For a given test agent, the CPDB may contain results for one or more experiments and for one or more species, strains, or routes of administration. For each carcinogen we select the most potent $\mathrm{TD}_{50}$ for a target site identified by the author of a published paper as reported in Gold et al. (1984). The range of $\mathrm{TD}_{50} \mathrm{~s}$ in the CPDB is more than 10 millionfold. For the correlation between mutagenic and carcinogenic potency we use the inverse of $\mathrm{TD}_{50}$, which has better statistical properties than the $\mathrm{TD}_{50}$ (Sawyer et al., 1984).

Mutagenic potency. We have used the initial slope of the Salmonella plate test dose-response curve as a measure of mutagenic potency (Bernstein et al., 1982). For a chemical tested in replicate experiments, the median slope of the positive experiments in the data-set was determined. For a chemical tested in replicate experiments under different protocols, the most potent median slope was used.

\section{Results}

(A) Use of color graphics to examine variability in Salmonella test data

Because Salmonella test data are derived from a variety of test conditions and laboratories and can be quite variable, methods of analysis must take advantage of trends. To be able to 'over-view' the information precisely would be useful, yet exceedingly difficult because of the volume of data.

Color graphics permit this kind of overview, as shown in Figs. 1 and 2 for selected chemicals. A number of variables characterizes each experiment; most are qualitative and define the protocol, and two are quantitative: potency and statistical significance. The parameters represented are: chemical; source of $S 9$ (the metabolic activation mixture, usually prepared from rodent liver); bacterial tester strain; laboratory; induction method; potency and $p$ value associated with the result; summary $p$ value if the experiment was part of a replicate set of experiments; and whether the slope of the dose-response curve was negative.

Fig. 1, which is six-dimensional, displays results from 30 chemicals under 1 test condition: hamster S9 from Aroclor-induced animals. These results are from the NCI/NTP Salmonella testing program cited above in which a chemical was tested by 4 different laboratories using a number of standardized protocols. The 30 were selected for illustrative purposes. Results in 5 tester strains are displayed. The color bar for each strain is divided into 4 parts to represent the 4 laboratories. A negative (i.e., statistically non-significant) experiment is indicated by a white ' $\mathrm{X}$ '. A potency (color) 

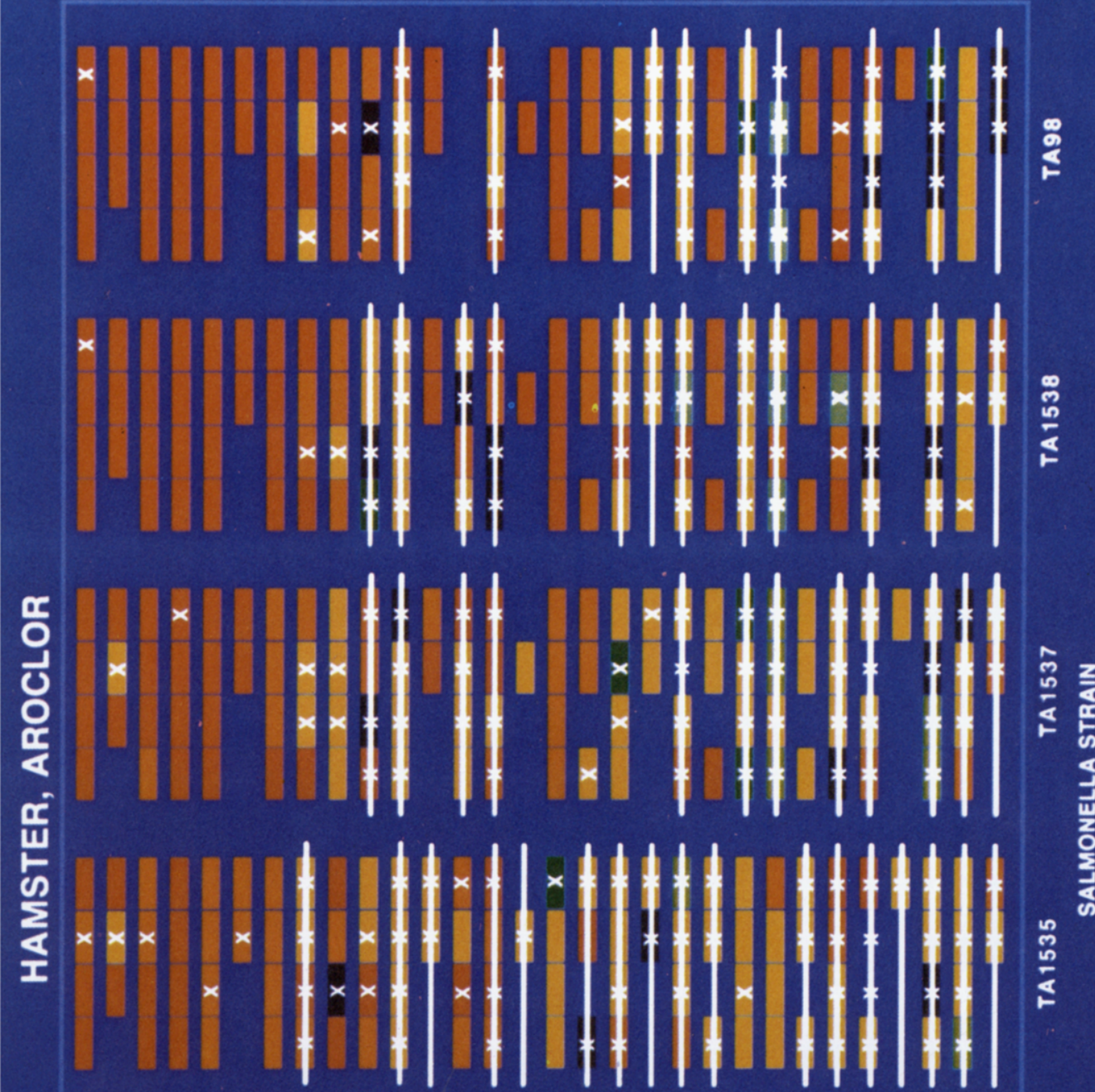

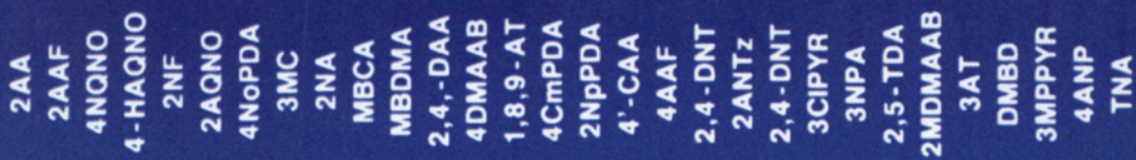

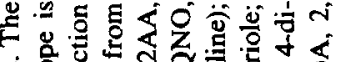

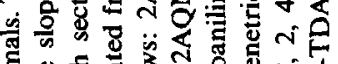

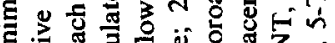

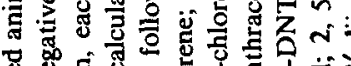

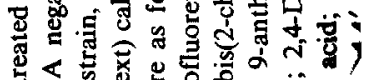

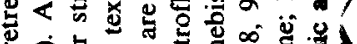

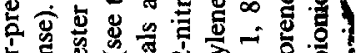

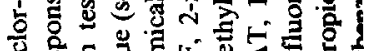

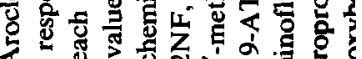

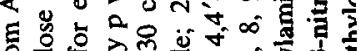
응 궁

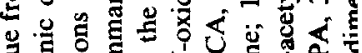

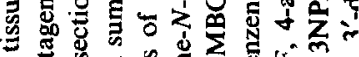

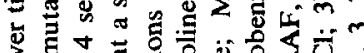

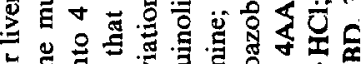

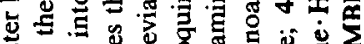

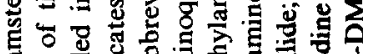

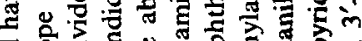

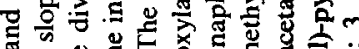

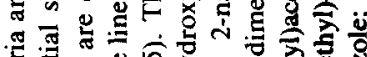

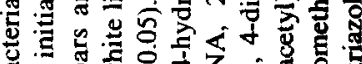

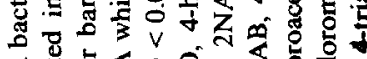

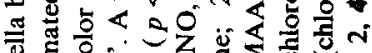

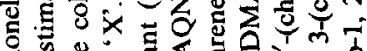

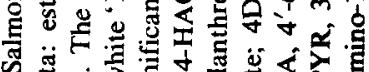

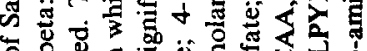
包药

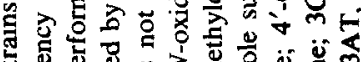

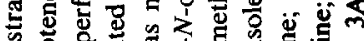

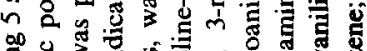

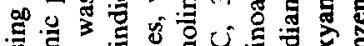

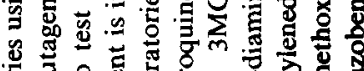

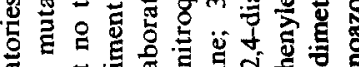

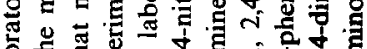

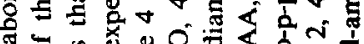

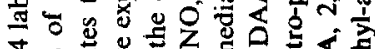

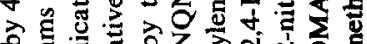

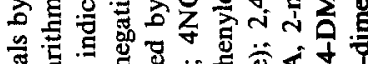

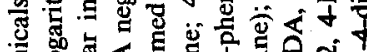

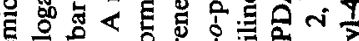

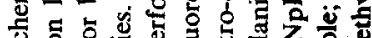
응영

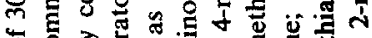
के वृ

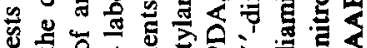

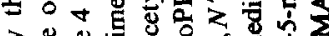

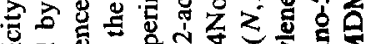

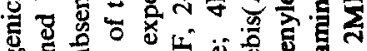

离

高

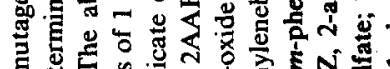

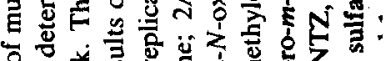

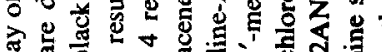

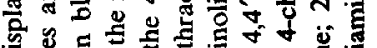

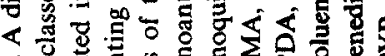

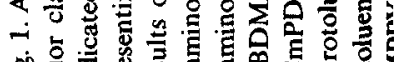
क응 


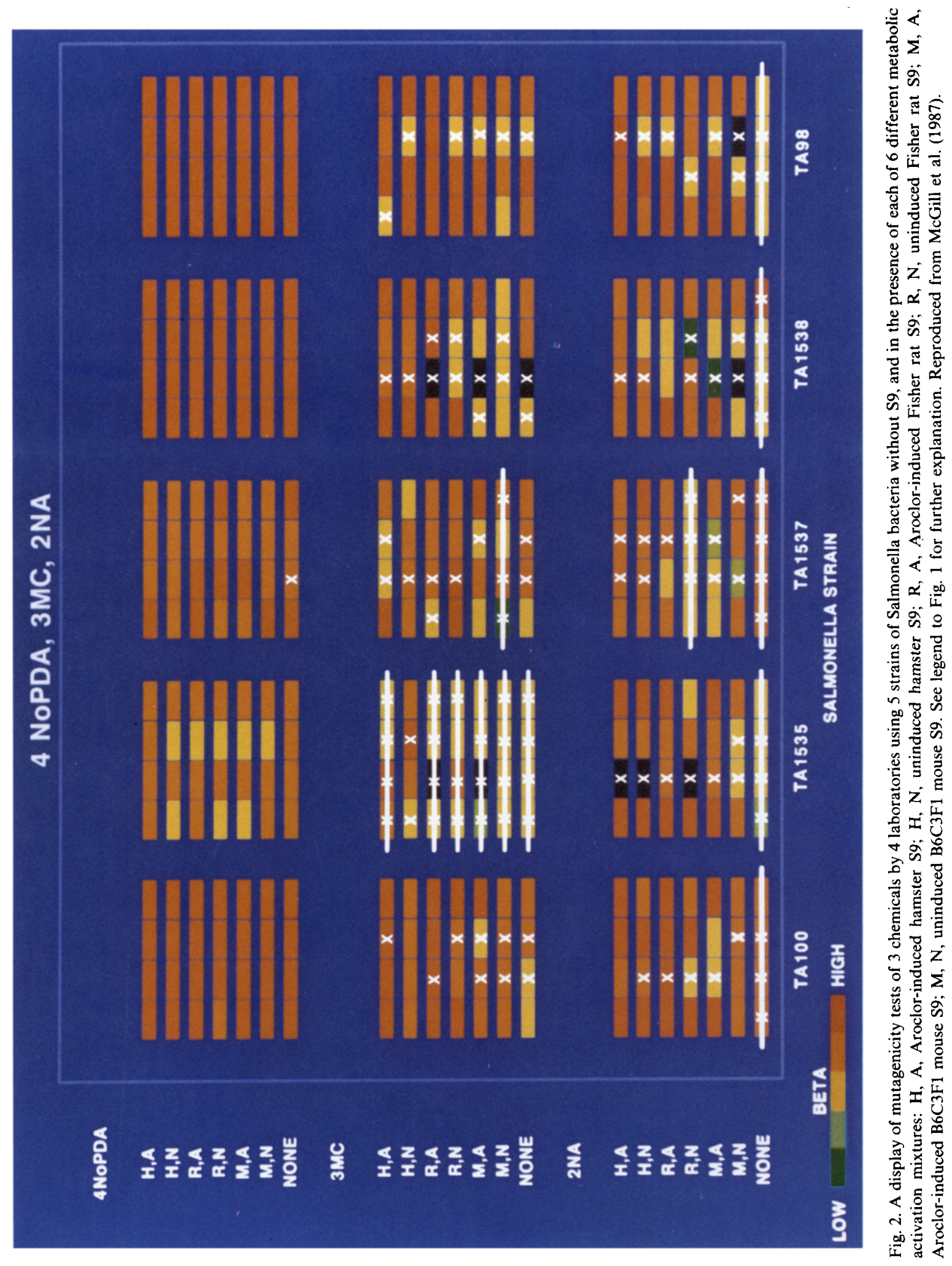


is indicated if the slope was positive, regardless of statistical significance. We use a white line to indicate that the data-set was non-significant overall. Note that though there is considerable disagreement among laboratories as to the positivity of individual experiments, there is considerable agreement as to potency, at least within the discrete categories we have defined. High potency results are generally uniform across laboratories, whereas results for chemicals of low potency vary substantially. Fig. 1 demonstrates that nearly all of the highly potent results are positive, while many of the low-potency results are not. This could suggest a limitation of the analytical procedure or a characteristic of the particular chemicals tested. Of possible interest in this regard is the observation that among the results of the NCI/NTP Salmonella testing program there is a significant relationship between potency and the number of tester strains that are reverted (Siegel, 1984).

Experiments with negative slopes are indicated in black. When these occur in replicate data-sets that are positive overall, they indicate mutagens for which the dose-response curve included a number of toxic doses, and the statistical procedure was not able to pick out the initial, positive portion of the response. For chemicals that produce non-significant results, half of the data-sets that are non-significant overall would be expected to have a negative slope by chance alone. Fewer than half have negative slopes in Fig. 1, which may reflect failure to fully compensate for the upward bias inherent in the statistical procedure (Berstein et al., 1982). Fig. 1 also indicates tester strain specificity for a number of chemicals (e.g., 3MC, 2NA).

Fig. 2 presents results for a variety of test conditions, and in only a few cases does source of S 9 or Aroclor induction affect positivity or potency (e.g., 3MC appears capable of reverting strain TA1535 only in the presence of uninduced hamster S9). This suggests it may be possible to combine multi-laboratory test data in which different activation systems have been used. This would be useful as it would greatly increase the number of chemicals and results that could be included in comparative analyses.
(B) Comparison of results from Salmonella tests and animal carcinogenesis bioassays

We compare: (1) positivity and (2) mutagenic and carcinogenic potencies for chemicals positive in both tests.

Positivity comparison. As shown in Table 1, 23 of the 48 compounds in the positivity analysis were evaluated by the NCI/NTP as clearly carcinogenic, and 8 were evaluated as suggestive of carcinogenicity. 37 of the 48 were mutagenic under at least 2 test conditions over the 20 Salmonella test protocols $(p<0.00256$, see Methods). Table $1 \mathrm{~A}$ indicates that when compounds are classified as positive only if the NCI/NTP evaluation was clear evidence of carcinogenicity, the sensitivity of the Salmonella test is quite high: $91 \%(21 / 23)$ of the carcinogens are also mutagens; however specificity is very low: only $36 \%(9 / 25)$ of the non-carcinogens are non-mutagens. Assuming the animal test results are correct, $63 \%$ of the mutagenesis results are accurate. The measure $\kappa$, which does not assume the accuracy of the animal cancer test results, is only 0.27 . On the right side of Table 1, a compound is classified as positive if the NCI/NTP evaluation was either clear or suggestive evidence of carcinogenicity. For this classification, specificity, accuracy, and $\kappa$ are higher, and sensitivity remains the same.

All but 1 (diphenylnitrosamine) of the $48 \mathrm{chem}$ icals from the NCI/NTP test programs we examined were included in the analysis of Dunkel et al. (1985). Dunkel et al. (1985) defined a Salmonella test as positive if there was "a dose-related increase with a least two doses being greater than, or equal to, two-fold background, unless the background was less than ten, in which case a three-fold increase was required". By their classification, 28 of the 47 chemicals were mutagens compared to 36 by our classification. All 19 chemicals that Dunkel et al. classified as non-mutagens, we also classified as non-mutagens. Of the 8 compounds that we classified as mutagens and they classified as non-mutagens, 3 are carcinogens (aniline $\mathrm{HCl} ; 4,4^{\prime}$-methylenebis $\left(N, N^{\prime}\right.$-dimethylaniline); reserpine) and 5 are non-carcinogens (acetohexamide; $N, N^{\prime}$-dicyclohexylthiourea; 
TABLE 1

3 COMPARISONS OF POSITIVITY IN SALMONELLA TESTS vs. RODENT CARCINOGENESIS TESTS ${ }^{\text {a }}$

\begin{tabular}{|c|c|c|c|c|}
\hline & \multicolumn{2}{|c|}{$\begin{array}{l}\text { Carcinogenicity including } \\
\text { suggestive evidence as negative }\end{array}$} & \multicolumn{2}{|c|}{$\begin{array}{l}\text { Carcinogenicity including } \\
\text { suggestive evidence as positive }\end{array}$} \\
\hline & + & - & + & - \\
\hline \multicolumn{5}{|l|}{ (A) Current analysis: 20 protocols } \\
\hline Mutagenicity + & 21 & 16 & 28 & 9 \\
\hline- & 2 & 9 & 3 & 8 \\
\hline $\begin{array}{l}\text { Sensitivity }=0.91 ; \text { specificity }=0.36 \\
\text { accuracy }=0.63 ; \kappa=0.27\end{array}$ & & & \multicolumn{2}{|c|}{$\begin{array}{l}\text { Sensitivity }=0.90 ; \text { speci- } \\
\text { ficity }=0.47 ; \text { accuracy }= \\
0.75 ; \kappa=0.41\end{array}$} \\
\hline \multicolumn{5}{|l|}{ (B) Analysis of Dunkel et al. ${ }^{\mathrm{b}}$} \\
\hline Mutagenicity + & 17 & 11 & 24 & 4 \\
\hline- & 5 & 14 & 6 & 13 \\
\hline $\begin{array}{l}\text { Sensitivity }=0.77 ; \text { specificity }=0.56 \\
\text { accuracy }=0.66 ; \kappa=0.33\end{array}$ & & & \multicolumn{2}{|c|}{$\begin{array}{l}\text { Sensitivity }=0.80 ; \text { speci- } \\
\text { ficity }=0.76 ; \text { accuracy }= \\
0.79 ; \kappa=0.55\end{array}$} \\
\hline \multicolumn{5}{|l|}{ (C) Current analysis: TA100/rat/Aroclor } \\
\hline Mutagenicity + & 20 & 9 & 26 & 3 \\
\hline- & 3 & 16 & 5 & 14 \\
\hline $\begin{array}{l}\text { Sensitivity }=0.87 ; \text { specificity }=0.64 \\
\text { accuracy }=0.75 ; \kappa=0.50\end{array}$ & & & \multicolumn{2}{|c|}{$\begin{array}{l}\text { Sensitivity }=0.84 ; \text { speci- } \\
\text { ficity }=0.82 ; \text { accuracy }= \\
0.83 ; \kappa=0.65\end{array}$} \\
\hline
\end{tabular}

a Test results are from NCI/NTP testing programs, as described in the text. Sensitivity, the proportion of carcinogens that are mutagens; specificity, the proportion of non-carcinogens that are non-mutagens; accuracy, the proportion of the correctly detected carcinogens and non-carcinogens to the total number of chemicals;, $2(a d-b c) /[(a+b)(b+d)+(c+d)(a+c)]$, where $a$ is the number of carcinogens that are mutagens; $b$ is the number of carcinogens that are non-mutagens; $c$ is the number of non-carcinogens that are mutagens; and $d$ is the number of non-carcinogens that are non-mutagens.

b Diphenylnitrosamine was not included in the data evaluated by Dunkel et al. (1985), and hence the total number of chemicals is 47 instead of 48 .

lithocholic acid; 1-phenyl-3-methyl-5-pyrazolone; and 2,3,5,6-tetrachloro-4-nitroanisole). These 8 chemicals were non-mutagenic by the criteria of Dunkel et al. because of the requirement that revertants increase by 2-3-fold. As shown in Table $1 \mathrm{~B}$, the classification method of Dunkel et al. results in accuracy similar to our rather elaborate statistical analysis, somewhat decreased sensitivity, and somewhat increased specificity.

We compared positivity in the carcinogenesis and mutagenesis tests for various subsets of the data (i.e., for each tester strain separately, and matching $\mathrm{S} 9$ source to the animal bioassay species and sex). In all but 1 case the associations are not greater than in Table. 1A. By far the greatest association is for TA100 with Aroclor-induced rat S9. As shown in Table 1C, when results for TA100 are compared to the values calculated using all tester strains, sensitivity using only TA100 is slightly lower, but the specificity is higher. The overall accuracy is much higher than when results across all 20 protocols are used.

Potency comparison. The correlation between carcinogenic and mutagenic potency for 80 carcinogens is plotted in Fig. 3. Carcinogenic potency $\left(1 / \mathrm{TD}_{50}\right)$ is plotted on the vertical axis. Mutagenic potency (the estimated initial slope of the mutagenic dose response) is plotted on the horizontal axis. (Pearson's correlation coefficient $=0.41, p=0.0002$; Spearman's rank correlation coefficient $=0.31, p=0.005$ ). When we included compounds considered by the NCI/NTP to be suggestive of carcinogenicity, the results are simi- 
lar: 9 chemicals are added to the analysis, Pearson's correlation coefficient $=0.37, p=0.0004$, Spearman's rank correlation coefficient $=0.30, p$ $=0.0047$.

The weakest and most potent mutagens are saccharin and aflatoxin $B_{1}$. When we remove these from the correlation, Pearson's correlation coefficient $=0.29, p=0.01$, and Spearman's rank correlation coefficient $=0.26, p=0.03$. The weakest

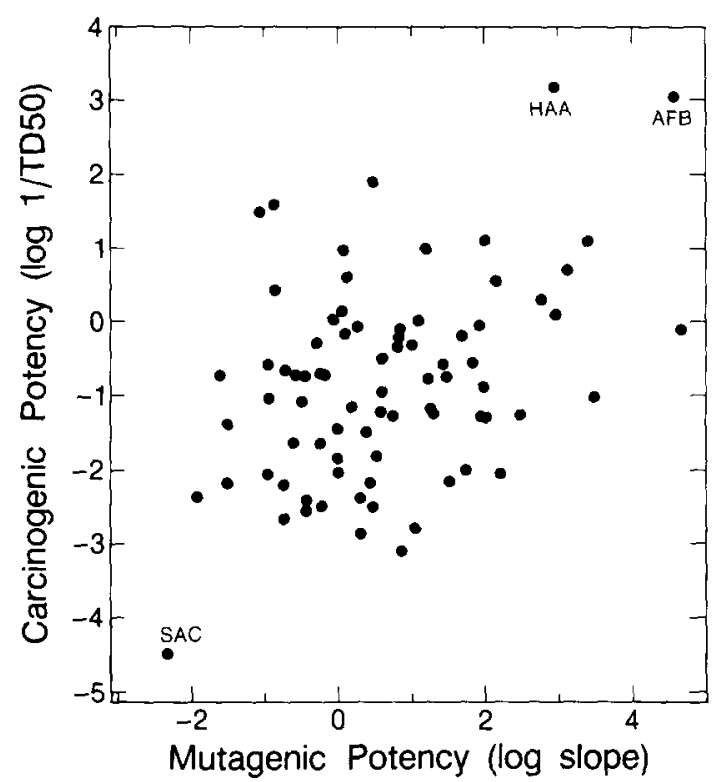

Fig. 3. Correlation of Carcinogenic and mutagenic potency. Carcinogenic potency is the inverse of the $\mathrm{TD}_{50}$ and mutagenic potency is the estimated initial slope of the mutagenic dose response. Abbreviations shown in the figure are: HAA, $N$-hydroxy-2-acetylaminofluorene; AFB, aflatoxin $\mathrm{B}_{1}$; SAC, saccharin. Chemicals plotted, and their carcinogenic and mutagenic potencies are, respectively: 2-acetylaminofluorene, 1.19, 949.2; 2-aminoanthraquinone, $0.01,56.0$; aflatoxin $B_{1}, 1070$, 37800; 3-amino-1,2,4-triazole, 0.04, 0.033; acronicine, 2.53, 0.10 ; aniline, 0.01, 34.5; 4-amino-2-nitrophenol, <0.003, 0.60; 2-amino-5-nitrothiazole, 0.02, 0.30; auramine, 0.09, 0.10; azobenzene, $0.05,5.7$; benzo[ $a]$ pyrene, $0.09,3100$; benzidine, $0.58,7.0$; direct dye black $38,1.06,0.90$; 4-chloro- $m$-phenylenediamine, $<0.003,3.0$; $m$-cresidine, $<0.002,0.20$; 4-chloro$o$-toluidine $\cdot \mathrm{HCl}, \quad 0.06,18.8 ;$ cupferron, $0.18 ; 0.30$; cyclophosphamide, $0.79,7.1 ; 7,12$-dimethylbenz $[a]$ anthracene, $11.9,103$; 4-dimethylaminoazobenzene, $0.30,4.0 ; 2$, 4-diaminoanisole, $0.01,166$; 1, 2-dibromoethane, 0.84, 1.9; 1,2-dibromo-3-chloropropane, 9.35, 16.1; 3,3'-dichlorobenzidine, $0.05,302$; dimethylcarbamyl chloride, $0.19,0.70$; diethylnitrosamine, 76.3, 3.10; diethylstilbestrol, 38.5, 0.10, diallate, $0.05,92.0$; dimethylnitrosamine, $8.85,1.20$; dinitrosopiperazine, $0.50,0.50$; ethylene imine, $3.53,149 ; \mathrm{N}$-hydroxy-2-ace- tylaminofluorene, 1450, 913; hydrazine sulfate, $0.45,6.7 ; 1^{\prime}$-hydroxysafrole, $0.08,0.30$; isophosphamide, $1.35,1.10 ; 3,3^{\prime}$-dimethoxybenzidine-4,4'-diisocyanate, $<0.001,7.60 ; 4,4^{\prime}$-methylenebis(2-chloroaniline), $0.11,4.00 ; 3$-methylcholanthrene, 4.95, 1380; metronidazole, <0.003, 0.40; Michler's ketone, $0.21,0.20 ; 4,4^{\prime}$-methylene-bis( $N, N^{\prime}$-dimethylaniline), 0.06 , 4.00; methyl methanesulfonate, $0.03,2.50$; 5-nitroacenaphthene, $0.13,101$; nitrilotriacetic acid (trisodium), <0.004, 0.012; 2naphthylamine, $0.05,107 ; 1,5$-naphthalenediamine, 0.01, 1.00; $N$-(4-(5-nitro-2-furyl)-2-thi ..., 0.76, 45400; nitrofen, 0.02, 3.5; $N$-methyl- $N^{\prime}$-nitro- $N$-nitrosoguanidine, $1.91,629 ; N$-nitrosopyrrolidine, $0.48,10.7 ; N$-nitrosopiperazine, $0.18,0.4 ; 5$-nitro$o$-toluidine, $<0.004,0.40 ; o$-anisidine, $0.04, \quad 1.00 ; o$ aminoazotoluene, $0.27,68.7 ; o$-phenylenediamine, $0.0040,1.97$; p-cresidine, $0.0224,0.59$; phenacetin, $0.0013,2.00$; phenylethylhydrazine sulfate, $0.0685,1.53 ; \beta$-propiolactone, $0.862,86.5$; $p$-quinone dioxime, $0.0094,1.01$; natulan, $3.95,1.39$; propane sultone, $0.275,68.3$; pivalolactone, $0.0065,2.82 ; N$-nitrosodiphenylamine, $0.0086,0.12$; reserpine, 29.2, 0.09; saccharin, $<0.00003,0.033$; sodium nitrite, $0.0065,0.03$; sterigmatocystin, $12.1,2600 ; 4,4^{\prime}$-thiodianiline, $0.179,31.2 ; 2,4$-diaminotoluene, $0.685,1.27$; toxaphene, $0.245,0.12$, tris-(2, 3-dibromopropyl)phosphate, $0.637,49.5$; vinyl chloride, $0.271,27.8$; sulfallate, $0.058,20.1 ; 1$, 2-dichloroethane, $0.182,0.02 ; 2$-nitro$p$-phenylenediamine, $0.0016,11.5$; 3-(chloromethyl)pyridine $\mathrm{HCl}, 0.0062,0.18$; 4-aminobiphenyl, 1.01, 12.9; 2, 4, 5-trimethylaniline, $0.163,17.0 ; 2,4$, 6-trimethylaniline, $0.193,0.58$.

and most potent carcinogens are saccharin and $N$-hydroxy-2-acetylaminofluorene. When we remove these from the correlation, Pearson's correlation coefficient $=0.33, p=0.003$, and Spearman's rank correlation coefficient $=0.26, p=0.02$. When all 3 extremes are removed, Pearson's correlation coefficient $=0.24, p=0.04$, and Spearman's rank correlation coefficient $=0.23, p=0.04$.

\section{Discussion}

Until recently, the predominant assumption in mutagenicity testing was that the Salmonella test may be capable of discriminating between carcinogens and non-carcinogens, but may not be capable of reflecting the relative potency of carcinogens. The work presented here suggests a more complex picture.

The overall accuracy (63-75\%: Table 1) of the mutagenesis test using a subset of the Dunkel et al. $(1984,1985)$ data-set is somewhat lower than our previous estimates (Ames and McCann, 1981). Sensitivity is somewhat greater but specificity is 
lower despite the use of the same batches of chemicals and rodent strains (for metabolic activation) in both mutagenesis and carcinogenesis assays. Below, we discuss these results in the context of previous work.

\section{Sensitivity}

Over a very large number of carcinogens, as reviewed by Rinkus and Legator (1979) and commented upon by Ames and McCann (1981), the sensitivity of the Salmonella assay is about $83 \%$. By our statistical analysis of the NCI/NTP dataset (Table 1) sensitivity is somewhat higher (about 90\%); by the analysis of Dunkel et al. (1985) it is somewhat lower (about 76\%). The greater sensitivity of the statistical analysis as compared to that of Dunkel et al. is expected since more than a 2-fold increase over background, as required by Dunkel et al., is not required for detection by the statistical procedure.

Overall, the results on positivity are consistent with the idea that a substantial fraction of chemical carcinogens are detected by the test. Bias in selection of compounds for testing is a confounding problem in assessing sensitivity of the assay. Chemicals selected for mutagenesis or carcinogenesis testing are not a random sample and it is complicated to account for possible biases. To the extent that selection of chemicals for testing has been guided by structural similarities to known mutagens, estimates of sensitivity will be biased upward. On the other hand, so many chemicals have now been tested including a large variety of chemical classes selected according to a variety of other criteria that such selection bias may be mitigated.

\section{Specificity}

High sensitivity does not mean much if the test does not distinguish carcinogens from noncarcinogens, and in results presented here, the specificity is low $(36-47 \%)$ (Table 1). This low specificity has also been noted by others (Shelby and Stasiewicz, 1984; Ashby and Purchase, 1985). Although there have been a number of studies comparing positivity in short-term tests other than the Salmonella assay and animal cancer tests, few non-carcinogens have been included in these studies and the range of chemical classes screened is much smaller than for the Salmonella system (see, for example, results compiled from the Gene-Tox Program (Haimes et al., 1985; Palajda and Rosenkranz, 1985; Mendelsohn, 1985)); therefore it is difficult to compare the performance of these to the Salmonella test. As results from the NTP short-term testing program become available, it should be possible to make more precise estimates of sensitivity and specificity of some of these other assays.

Almost all of the non-carcinogens in the Dunkel et al. data-set that are mutagenic are weak mutagens - i.e., they require a relatively high dose to elicit an effect. (This was also observed previously for a different set of compounds (McCann et al., 1975, 1976).) Perhaps this is a consequence of differences in the sensitivities of Salmonella and rodents to overall toxic effects. Taking the whole genome into account, mutagenesis due to a particular compound might occur over a very wide dose range. However, since mutagenesis tests target only a small fraction of the genome, a positive mutagenic effect can only be detected at high, near-toxic doses. If the MTD in a rodent for a particular compound occurs at low doses relative to the genotoxic potency in the rodent, then the carcinogenesis bioassay may be negative whereas the Salmonella test may be positive if the predominant toxic effect in Salmonella is genotoxicity. The converse may also be expected to occur: carcinogens might not be detected as mutagens because of non-genotoxic toxicity to Salmonella. For example, the mutagenic effects of an antibiotic that kills bacteria at low doses by interfering with the growth of cell walls would go undetected in the Salmonella assay; however, it might be detected in a mutagenesis assay using rodent cells, or as a carcinogen in rodents. This would result in assays of 'false negatives'. Given the greater complexity of the mammalian system, it is not unreasonable to expect that this phenomenon might result in more 'false positives' than 'false negatives'.

\section{Potency}

The evidence presented here and in the general literature suggests that there may be a weak correlation between mutagenic potency estimated from the DNA-damaging short-term tests and carcino- 
genic potency. The analysis presented here includes the largest number of chemicals examined thus far $(80)$, and there is a weak correlation between potencies (Fig. 3: $r=0.41$ ). Earlier, Parodi et al. (1983) examined this relationship for 59 chemicals. Using Salmonella and animal cancer test potencies estimated from published data, they reported a significant correlation of 0.34 . We investigated the relationship for the 26 chemicals from the Parodi analysis for which we have mutagenic and carcinogenic potency values. The correlation for these chemicals was similar to their published results. Meselson and Russell (1977) reported a very high correlation $(r=0.92)$ for 9 non-nitroso compounds using Salmonella test results of McCann et al. (1975) and animal cancer test potencies they estimated from published results. Our analysis of these same compounds produced a lower but still strong correlation, $r=0.70$. Numerous other studies have examined small data-sets, usually for chemicals related in structure (e.g., Okano et al., 1979; Coombs et al., 1980; Wood et al., 1980; Scribner et al., 1980; Bartsch et al., 1980; Parodi et al., 1982; Hsieh et al., 1977; Ashby et al., 1982; Zeiger and Sheldon, 1978a, b; Rao et al., 1977, 1979; Lee and Guttenplan, 1981). The number of compounds analyzed and the results of these analyses vary widely. Though it would be of interest to try to bring together this work into an overall analysis, it would be complex to incorporate these diverse studies into a standardized format permitting joint analysis.

Other researchers have examined the potency relationship using results from other short-term tests. Correlations have been reported for the mouse lymphoma assay (Clive, 1977), covalent DNA binding (Lutz, 1979), and in vitro transformation (Parodi et al., 1983).

We have used the reported values for mutagenic potency in other short-term tests to examine the relationship to our carcinogenic potency values. Although the number of substances included in these analyses is quite small, the Gene-Tox reports provide the best available data. Using the most potent values reported for each chemical, for sister-chromatid exchange (Latt et al., 1981) we found no correlation in potencies for either in vitro results $(N=28)$ or in vivo results $(N=11)$; for the micronucleus assay (Heddle et al., 1983) we found $r=0.66, N=24$; and for the sperm abnormality assay (Wyrobek et al., 1983) we found $r=0.92, \quad N=17$. Though these results suggest there may be a correlation for some short-term tests, without further analysis it is not possible to draw any definitive conclusions.

The work of Bernstein et al. (1985a, b), Zeise et al. (1984) and Crouch et al. (1987) may be relevant to the correlation in potency. These groups found a correlation between acute (Zeise et al., 1984) or chronic (Bernstein et al., 1985) toxicity and carcinogenic potency. Bernstein et al. pointed out that, in the absence of $100 \%$ tumor incidence in treated animals, the potency of a carcinogen is restricted to about a 30 -fold range about the maximum dose tested in a standard carcinogenesis bioassay and that this, together with the wide range of MTDs tested, provides a statistical basis for the correlations reported. They also suggest a biological explanation for the correlation:

If some compounds were highly carcinogenic compared with their MTDs, then we would expect to observe $100 \%$ (or at least very high) incidence rates at all of the experimental dose levels. This was not seen with the compounds under study. If the saturation of a metabolic activation process was involved, the dose response might plateau. From our database we observed that approximately $10 \%$ of the dose-response functions were sublinear, indicating possible saturation. For the compounds in which this was observed, it was, however, generally not replicated in other target sites in the same experiment, in the other sex of the same species, or in other species.

These findings may be important for interspecies extrapolation. They are consistent with the hypothesis that cell death and consequent cell proliferation may be required for carcinogenesis (see for example, Farber, 1984). This would challenge the reasonableness of low dose risk extrapolation to humans from high dose animal cancer tests (Ames et al., 1987).

The results we have reported here suggest several relevant hypotheses relating to interspecies extrapolation.

(1) The positivity association was strongest for TA100 alone (Table 1), and only in this strain does mutagenesis occur predominantly via an SOS type of error-prone repair mechanism. This type of repair mechanism is also present in mammalian cells, and normally functions at a very low rate unless the system is stressed by toxicity to the DNA, in which case it is induced. Thus, one might 
hypothesize that if SOS-mediated mutagenesis were most relevant to carcinogenesis, such a process might be linked to toxicity at the level of DNA.

(2) If some toxicity at the level of DNA is required, either to induce an error-prone repair system or simply to generate a high enough mutation rate to detect mutagenesis at the histidine operon in Salmonella or carcinogenesis in the rodent bioassays, a correlation between carcinogenic and mutagenic potencies might not be unexpected. Though Salmonella and rodents are far apart on the evolutionary scale, the basic structure of DNA in the two is the same, and the general types of repair systems are similar. It might therefore be of interest to compare the overall correlation in toxicity between the two systems for chemicals that are detected as both carcinogens and mutagens, and for chemicals which are mutagenic but not carcinogenic.

A great deal of effort continues to be expended to design batteries of short-term tests, which, taken together, might reliably indicate carcinogenic potential. A recent approach has been to evaluate test batteries by combining sensitivities and specificities determined from validation studies of the individual tests (e.g., Chankong et al., 1985; Benigni and Giuliani, 1985; Ennever and Rosenkranz, 1986). Though the use of such methods may give important insights, they may be difficult to generalize. The larger the number of short-term tests in a test battery, the greater the number of ways one can permute the decision rules. It may therefore be possible to design a set of decision rules that will suggest a high level of predictive accuracy for a particular set of chemicals but which will not be predictive for other chemicals. Attention must be given to choosing complementary tests based on knowledge of why certain chemicals are false positives or negatives. Ashby and Purchase (1985) have begun to address these questions for false positives in the Salmonella assay.

The primary difficulty in examining the relevance of short-term tests to effects in humans is the paucity of epidemiologic data on carcinogenesis. Furthermore, the individuals in most epidemiologic studies are exposed at high doses, e.g., occupational exposures, whereas most individuals are exposed at much lower doses. Animal cancer tests, though they offer the advantage of being numerous and available, are conducted at doses that approximate the MTD. Thus, though a relationship between results in short-term tests and carcinogenesis tests may be demonstrated, its meaning is unclear because both assays are conducted at high doses.

\section{Acknowledgements}

Supported by NIEHS/DOE Interagency, Agreement 222-4Y01-10066.

We acknowledge the assistance of Renae Magaw.

\section{References}

Ames, B.N., and J. McCann (1981) Validation of the Salmonella test: a reply to Rinkus and Legator, Cancer Res., 41, 4192-4203.

Ames, B.N., R. Magaw and L.S. Gold (1987) Ranking possible carcinogenic hazards, Science, 236, 271-280.

Ashby, J., P.A. Lefevre, J.A. Styles, J. Charlesworth and D. Paton (1982) Comparisons between carcinogenic potency and mutagenic potency to Salmonella in a series of derivatives of 4-dimethylaminoazobenzene (DAB), Mutation Res., $93,67-81$.

Ashby, J., and I.F.H. Purchase (1985) Significance of the genotoxic activities observed in vitro for 35 of 70 NTP noncarcinogens, Environ. Mutagen., 7, 747-758.

Bartsch, H., C. Malaveille, A.M. Camus, G. Martel-Planche, G. Brun, A. Hautefeuille, N. Sabadie, A. Barbin, T. Kuroki, C. Frevon, C. Piccoli and R. Montesano (1980) Bacterial and mammalian mutagenicity tests: Validation and comparative studies on 180 chemicals, in: R. Montesano, H. Bartsch and L. Tomatis (Eds.), Cellular Aspects of Carcinogen Screening Tests, IARC, Lyon.

Benigni, R., and A. Giuliani (1985) Cluster analysis of shortterm tests: A new methodological approach, Mutation Res., $147,139-151$.

Bernstein, L., J. Kaldor, J. McCann and M.C. Pike (1982) An empirical approach to the statistical analysis of mutagenesis data from the Salmonella test, Mutation Res., 97, 267-281.

Bernstein, L., L.S. Gold, B.N. Ames, M.C. Pike and D.G. Hoel (1985a) Some tautologous aspects of the comparison of carcinogenic potency in rats and mice, Fund. Appl. Toxicol., 5, 79-86.

Bernstein, L., L.S. Gold, B.N. Ames, M.C. Pike and D.G. Hoel (1985b) Toxicity and carcinogenic potency, Risk Anal., 5, 263-264.

Chankong, V., Y.Y. Haimes, H.S. Rosenkranz and J. Pet-Edwards (1985) The carcinogenicity prediction and battery selection (CPBS) method: A Bayesian approach, Mutation Res., 153, 135-166. 
Clive, D. (1977) A linear relationship between tumorigenic potency in vivo and mutagenic potency at the heterozygous thymidine kinase $(\mathrm{TK}+/-)$ locus of L5178Y mouse lymphoma cells coupled with mammalian metabolism, in: D. Scott, B.A. Bridges and F.H. Sobels (Eds.), Progress in Genetic Toxicology, Elsevier, Amsterdam, pp. 241-247.

Coombs, M.M., T.S. Bhatt, A.M. Kissonerghis and C.W. Vose (1980) Mutagenic and carcinogenic metabolites of the carcinogen 15, 16-dihydro-11-methylcyclopenta[ $a$ ]phenanthrene-17-one, Cancer Res., 40 882-886.

Crouch, E., R. Wilson and L. Zeise (1987) Tautology or not tautology? J. Toxicol. Environ. Health, 20, 1-10.

Dunkel, V.C., E. Zeiger, D. Brusick, E. McCoy, D. McGregor, K. Mortelmans, H.S. Rosenkranz and V.F. Simmon (1984) Reproducibility of microbial mutagenicity assays, I. Tests with Salmonella typhimurium and Escherichia coli using a standardized protocol, Environ. Mutagen., 6, Suppl. 2, $1-251$.

Dunkel, V.C., E. Zeiger, D. Brusick, E. McCoy, D. McGregor, K. Mortelmans, H.S. Rosenkranz and V.F. Simmon (1985) Reproducibility of microbial mutagenicity assays, II. Testing of carcinogens and noncarcinogens in Salmonella typhimurium and Escherichia coli, Environ. Mutagen., 7, Suppl. 5, 1-248.

Ennever, F.K., and H.S. Rosenkranz (1986) Short-term test results for NTP noncarcinogens: An alternate, more predictive battery, Environ. Mutagen., 8, 849-865.

Farber, E. (1984) Cellular biochemistry of the stepwise development of cancer with chemicals: G.H.A. Clowes Memorial Lecture, Cancer Res., 44, 5463-5474.

Fisher, R.A. (1958) Statistical Methods for Research Workers, 13th Edn., Hafner, New York, p. 91.

Gold, L.S., C.B. Sawyer, R. Magaw, G.M. Backman, M. de Veciana, R. Levinson, N.K. Hooper, W.R. Havender, L. Bernstein, R. Peto, M.C. Pike and B.N. Ames (1984) A carcinogenic potency database of the standardized results of animal bioassays, Environ. Health Perspect., 58, 9-319.

Gold, L.S., M. deVeciana, G.M. Backman, R. Magaw, P. Lopipero, M. Smith, M. Blumenthal, R. Levinson, L. Bernstein and B.N. Ames (1986) Chronological supplement to the carcinogenic potency database: standardized results of animal bioassays published through December 1982, Environ. Health Perspect., 67, 161-200.

Gold, L.S., T.H. Slone, G.M. Backman, R. Magaw, M. DaCosta, P. Lopipero, M. Blumenthal and B.N. Ames (1987) Second chronological supplement to the carcinogenic potency database: Standardized results of animal bioassays published through December 1984 and by the National Toxicology Program through May 1986, Environ. Health Perspect., 74, 237-329.

Haimes, Y.Y., V. Chankong, J. Pet-Edwards, M. Palajda and H.S. Rosenkranz (1985) The carcinogenicity prediction and battery selection method: Theory and application, Mutation Res., 153, 79-200.

Heddle, J.A., M. Hite, B. Kirkhart, K. Mavournin, J.T. MacGregor, G.W. Newell and M.F. Salamone (1983) The induction of micronuclei as a measure of genotoxicity: A report of the U.S. Environmental Protection Agency GeneTox program, Mutation Res., 123, 61-118.
Hollstein, M., J. McCann, F. Angelosanto and W. Nichols (1979) Short-term tests for carcinogens and mutagens, Mutation Res., 65, 133-226.

Horn, L., J. Kaldor and J. McCann (1983) A comparison of alternative measures of mutagenic potency in the Salmonella (Ames) test, Mutation Res., 109, 131-141.

Hsieh, E.P.H., J.J. Wong, Z.A. Wong, C. Michas and B.H. Ruebner (1977) Hepatic transformation of aflatoxin and its carcinogenicity, in: H.H. Hiatt, J.D. Watson and J.A. Winsten (Eds.), Origins of Human Cancer, Book C, Cold Spring Harbor Press, Cold Spring Harbor, NY, pp. 697-707.

IARC (1980) Long-term and short-term screening assays for carcinogens: A critical appraisal, IARC Monographs on the Evaluation of the Carcinogenic Risk of Chemicals to Humans, Suppl. 2, IARC, Lyon.

Latt, S.A., J. Allen, S.E. Bloom, A. Carrano, E. Falke, D. Kram, E. Schneider, R. Schreck, R. Tice, B. Whitfield and S. Wolff (1981) Sister-chromatid exchanges: A report of the Gene-Tox program, Mutation Res., 87, 17-62.

Lee, S.Y., and J.B. Guttenplan (1981) A correlation between mutagenic and carcinogenic potencies in a diverse group of $N$-nitrosamines: Determination of mutagenic activities of weakly mutagenic $N$-nitrosamines, Carcinogenesis, 2, $1339-1344$

Lutz, W.K. (1979) In vivo covalent binding of organic chemicals to DNA as a quantitative indicator in the process of chemical carcinogenesis, Mutation Res., 65, 289-356.

McCann, J., and B.N. Ames (1976) Detection of carcinogens as mutagens in the Salmonella/microsome test: Assay of 300 chemicals: Discussion, Proc. Natl. Acad. Sci. (U.S.A.), 73, 950-954.

McCann, J., E. Choi, E. Yamasaki and B.N. Ames (1975) Detection of carcinogens as mutagens in the Salmonella/microsome test: Assay of 300 chemicals, Proc. Natl. Acad. Sci. (U.S.A.), 72, 5135-5139.

McCann, J., L. Horn and J. Kaldor (1984) An evaluation of Salmonella (Ames) test data in the published literature: Application of statistical procedures and analysis of mutagenic potency, Mutation Res., 134, 1-47.

McGill, R., T.E. Graedel and J. McCann (1988) A picture worth 1350 numbers, IEEE Comput. Graph. Applic., in press.

Mendelsohn, M.L. (1985) Can chemical carcinogenicity be predicted by short-term tests? Paper prepared for submittal to the 5th International Conference on Occupational and Environmental Significance of Industrial Carcinogens, October 8-10, 1985, Bologna, Italy.

Meselson, M., and K. Russell (1977) Comparisons of carcinogenic and mutagenic potency, in: $\mathrm{H}$. Hiatt, J. Watson and J. Winsten (Eds.), Origins of Human Cancer, Cold Spring Harbor Laboratory, New York, pp. 1473-1481.

Okano, T., T. Horie, T. Koike and N. Motohashi (1979) Relationship of carcinogenicity, mutagenicity, and K-region reactivity in benz [ $c$ ]acridines, Gann, 70, 749-754.

Palajda, M., and H.S. Rosenkranz (1985) Assembly and preliminary analysis of a genotoxicity data base for predicting carcinogens, Mutation Res., 153, 79-134.

Parodi, S., M. Taningher, P. Boero and L. Santi (1982) Quantitative correlations amongst alkaline DNA fragmen- 
tation, DNA covalent binding, mutagenicity in the Ames test and carcinogenicity, for 21 compounds, Mutation Res., 93, 1-24.

Parodi, S., M. Taningher, P. Russo, M. Pala, D. Vecchio, G. Fassina and L. Santi (1983) Quantitative predictivity of the transformation in vitro assay compared with the Ames test, J. Toxicol. Environ. Health, 12, 483-510.

Peto, R., M.C. Pike, L. Bernstein, L.S. Gold and B.N. Ames (1984). A proposed general convention for the numerical description of the carcinogenic potency of chemicals in chronic-exposure animal experiments, Environ. Health Perspect., $58,1-8$.

Rao, T.K., A.A. Hardigree, J.A. Young, W. Lijinsky and J.L. Epler (1977), Mutagenicity of $N$-nitrosopiperidines with Salmonella typhimurium/microsomal activation system, Mutation Res., 56, 131-145.

Rao, T.K., D.W. Ramey, W. Lijinsky and J.L. Epler (1979) Mutagenicity of cyclic nitrosamines in Salmonella typhimurium: effect of ring size, Mutation Res., 67, 21-26.

Sawyer, C., R. Peto, L. Bernstein and M.C. Pike (1984) Calculation of carcinogenic potency from long-term animal carcinogenesis experiments, Biometrics, 40, 27-40.

Scribner, N.K., B. Woodworth, G.P. Ford and J.D. Scribner (1980) The influence of molecular size and partition coefficients on the predictability of tumor initiation in mouse skin from mutagenicity in Salmonella typhymurium, Carcinogenesis, 1, 715-719.

Shelby, M.D., and S. Stasiewicz (1984) Chemicals showing no evidence of carcinogenicity in long-term, two-species rodent studies: The need for short-term test data, Environ. Mutagen., 6, 871-876.

Siegel, R.D. (1984) Characterization of the effects of test protocol and non-systematic variability in the Salmonella mutagenesis assay, Ph.D. Thesis, submitted to Univ. of Colorado.

Wood, A.W., W. Levin, R.L. Chang, M.T. Huang, D.E. Ryan, P.E. Thomas, R.E. Lehr, S. Kumar, M. Koreeda, H. Akagi, Y. Ittah, P. Dansette, H. Yagi, D.M. Jerina and A.H. Conney (1980) Mutagenicity and tumor-initiating activity of cyclopenta[ $c, d]$ pyrene and structurally-related compounds, Cancer Res., 40, 642-649.

Wyrobek, A.J., L.A. Gordon, J.G. Burkhart, M.W. Francis, R.W. Kapp Jr., G. Letz, H.V. Malling, J.C. Topham and M.D. Whorton (1983) An evaluation of the mouse sperm morphology test and other sperm tests in nonhuman mammals: A report of the U.S. Environmental Protection Agency Gene-Tox Program, Mutation Res., 115, 1-72.

Zeiger, E., and A.T. Sheldon (1978a) The mutagenicity of $N$-nitrosopiperidines for Salmonella typhimurium, Mutation Res., 57, 85-89.

Zeiger, E., and A.T. Sheldon (1978b) The mutagenicity of heterocyclic $N$-nitrosamines for Salmonella typhimurium, Mutation Res., 57, 1-10.

Zeise, L., R. Wilson and E. Crouch (1984), Use of acute toxicity to estimate carcinogenic risk, Risk Anal., 4, $187-199$. 